\title{
AUTOSOMAL RECESSIVE POLYCYSTIC KIDNEY DISEASE WITH CONGENITAL HEPATIC FIBROSIS- A CASE REPORT
}

\author{
Kangkana Mahanta1, Pronami Borah², Karabi Bora ${ }^{3}$
}

${ }^{1}$ Senior Resident, Department of Radiodiagnosis, Guwahati Neurological Research Centre, Guwahati, Assam, India.

${ }^{2}$ Associate Professor, Department of Radiodiagnosis, Assam Medical College and Hospital, Assam, India.

3Postgraduate Trainee, Department of Radiodiagnosis, Assam Medical College and Hospital, Assam, India.

HOW TO CITE THIS ARTICLE: Mahanta K, Borah P, Bora K. Autosomal recessive polycystic kidney disease with congenital hepatic fibrosis- a case report. J. Evolution Med. Dent. Sci. 2018;7(44):4821-4824, DOI: 10.14260/jemds/2018/1074

\section{PRESENTATION OF CASE}

A 11 years old female child presented with pain abdomen and abdominal distension on and off ever since she was 4 years; not associated with vomiting or altered bowel habits. There was no history of fever, dysuria or increased frequency of micturition. She also had decreased growth rate along with impairment of cognition and memory. The child had a history of one episode of hematemesis when she was 9 months old. There was no history of yellowish discoloration of the skin or urine. She was born spontaneously by vaginal delivery and the neo-natal period was uneventful. There was no medically significant family history of any illness running in her family in the previous two generations.

The child had pallor on clinical examination. Her blood pressure was within normal limits. She was short statured. Per-abdominally, liver was palpable, and the spleen was $4 \mathrm{~cm}$ below the left sub-costal margin. Her jerks were exaggerated. The laboratory parameters showed normal liver and renal function tests. Her serum TSH level was >100 IU.

\section{CLINICAL DIAGNOSIS}

Hypothyroidism with Hepato-Splenomegaly with Features of Encephalopathy.

\section{RADIOLOGICAL DIAGNOSIS}

She presented to department of Radiodiagnosis for USG whole abdomen which revealed mild hepatomegaly with enlargement of the caudate lobe (Fig. 1 A). The hepatic echotexture was mildly coarsened with regular surface. There was cystic dilatation of the intra-hepatic biliary radicals in Segment VII of the liver (Fig. 1B). Splenomegaly with dilated splenic vein was noted. The portal vein showed cavernous transformation (Fig. 1C) along multiple peri-pancreatic, splenic hilar and splenorenal collaterals. There was periportal echogenicity, suggestive of peri-portal fibrosis.

Bilateral kidneys were increased in size measuring $12.2 \mathrm{x}$ $5 \mathrm{~cm} \& 12.5 \times 5.6 \mathrm{~cm}$ respectively on right and left side. The renal cortical echotexture and thickness were well maintained. There was increased echogenicity of the renal pyramids along with multiple hyperechoic foci casting posterior acoustic shadow within the areas of tubular dilatation (Fig. 2 A).

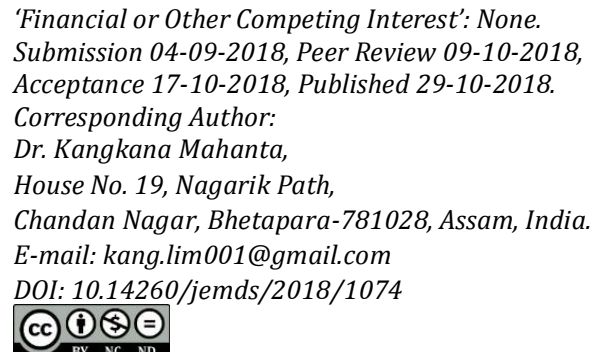

The child also underwent USG of the thyroid gland. Both the lobes and isthmus of the thyroid gland were very small in size, the anterior-posterior dimensions of the right and left lobes measuring $6 \mathrm{~mm}$ and $5.8 \mathrm{~mm}$ respectively. The glandular echotexture was altered but with normal vascularity on Colour Doppler Study. Few cystic lesions were noted in both the lobes of the small thyroid gland.

MRI of the abdomen showed altered hepatic morphology with enlarged caudate and left lobes. There was mild dilatation of the right lobar intra-hepatic biliary radicles in Segment VI and VII. CBD was normal in course and caliber. Post-contrast scan revealed normal hepatic enhancement. Portal vein showed cavernous transformation (Fig. 3A) without evidence of any obstructive pathology, along with dilatation and tortuosity of the superior mesenteric vein. There were multiple oesophageal and para-rectal varices (Fig. 3B), peri-pancreatic, gastro-hepatic, gastro-splenic, splenorenal, splenic hilar, retro-pancreatic and mesenteric collaterals. The spleen was enlarged in size $(13 \mathrm{~cm})$ along with a dilated splenic vein. Bilateral kidneys were enlarged in size and showed striated nephrogram.

She also underwent MRI brain. There was evidence of symmetrical T1 hyperintensities in bilateral globi pallidi and substantia nigra showing subtle restriction on DWI. MRspectroscopy revealed glutamine/glutamate peak between 2.2 and $2.4 \mathrm{ppm}$. The pituitary gland was normal in dimension with normal post-contrast enhancement. TOF angiogram revealed normal appearance of the major intracranial vessels without evidence of any aneurysm.

The child was diagnosed as a case of juvenile form of ARPKD complicated by portal hypertension without renal functional impairment and was taken up for Trans-jugular Intra-hepatic porto-systemic shunt.

\section{DIFFERENTIAL DIAGNOSIS}

1. Extrahepatic Portal Venous Obstruction with Portal Cavernoma Cholangiopathy.

2. Autosomal Dominant Polycystic Kidney Disease.

ARPKD is a heritable but phenotypically variable disorder characterized by non-obstructive fusiform ectasia of the renal collecting ducts leading to enlarged spongiform kidneys and ductal plate malformation of the liver resulting in congenital hepatic fibrosis. ARPKD/CHF has a broad spectrum of clinical presentations involving the kidney and liver. Imaging plays an important role in the diagnosis and follow-up of ARPKD. Conventional and high-resolution US and MRCP allow in defining of the extent of kidney and hepatobiliary manifestations. We present a case of a 11-year-old female child with ARPKD with congenital hepatic fibrosis. 
Autosomal recessive polycystic kidney disease is a phenotypically variable disorder, with an estimated incidence of 1 in 20, 000 live births, and characterized by varying degrees of non-obstructive renal collecting duct and hepatic biliary duct ectasia and fibrosis of both liver and kidneys. The severity of renal and hepatic disease are inversely proportional.(1) The most typical disease expression in neonates includes a history of oligohydramnios, massively enlarged kidneys, and the "Potter" sequence with pulmonary hypoplasia that leads to respiratory insufficiency and perinatal death. The typical renal phenotype of ARPKD consists of enlarged, echogenic kidneys with fusiform dilatation of the collecting ducts. (2) Patients with ARPKD also have liver involvement with increased number of bile ducts which are dilated. Persistence of the immature ductal elements simulates the formation of peri-portal fibrosis,(3) a pattern referred to as congenital hepatic fibrosis (CHF) and is always present in ARPKD. The most troublesome sequelae of ARPKD is portal hypertension and cholangitis due to the periportal fibrosis. These patients are at increased risk of benign and malignant hepatic tumours, especially cholangiocarcinoma.(4)

Blythe and Ockenden, in 1971, categorized the spectrum of ARPKD into four distinct groups-perinatal, neonatal, infantile, and juvenile. The most severe involvement occurs in the peri-natal group, and is characterized by huge kidneys and severe respiratory and renal compromise with death occurring within the 1 st week of life.

The neonatal group presents in the 1st month of life with approximately $60 \%$ duct involvement and death within their $1^{\text {st }}$ year.

The infantile form of ARPKD, presenting at age 3-6 months, is characterized by approximately $25 \%$ duct involvement and development of both renal failure and portal hypertension.

The juvenile form manifests the least renal disease (About $10 \%$ duct involvement) and presents between the ages of 6 months and 5 years with portal hypertension.

\section{Genetic Background of ARPKD}

ARPKD is caused by mutations in PKHD1, located on chromosome 6p21.1-p12. The product of PKHD1, fibrocystin/ polyductin (FPC),(5) is expressed predominantly in the kidney, liver and pancreas. Dysfunction of fibrocystin leads to abnormal ciliary signalling, which is normally required for regulation of proliferation and differentiation of renal and biliary epithelial cells.

\section{Renal Manifestations/Clinical Features}

The collecting ducts are dilated and elongated in ARPKD, in association with interstitial fibrosis resulting in renal function impairment. Most patients with ARPKD proceed to end stage renal disease (ESRD) and the later the age at initial manifestations, the later the development of ESRD.

Systemic hypertension is also a manifestation of many patients with ARPKD. These children are prone to develop frequent urinary tract infection.

\section{Radiological Features}

Abdominal distention is evident on radiographs, and gasfilled bowel loops are often deviated centrally. With severe kidney disease, the baby may be born with pulmonary hypoplasia and a small thorax.

At sonography, the kidneys are enlarged and echogenic and continues to grow in the first 2-3 years of life and then plateaus or even decreases in size, unlike in ADPKD, where there is progressive growth throughout their lives. There may be loss of the cortico-medullary differentiation and the cortex may appear as a thin peripheral hypoechoic rim. There is a spectrum of altered echogenicity of the renal pyramids, due to variable degrees of tubular dilatation, cyst formation, and hyperechoic foci of crystal deposition(6) that may represent urine stagnation within dilated tubules including calcium precipitation secondary to diminished citrate excretion. This feature was seen in our patient along with bilateral nephromegaly.

At unenhanced computed tomography (CT), the kidneys are smooth, enlarged, and low in attenuation, due to the large fluid volume in the dilated ducts. On post-contrast scans, the kidneys show a striated nephrogram which represents accumulation of contrast material in the dilated tubules. If renal function is considerably impaired, there may be poor opacification and excretion.

On MRI, the enlarged kidneys are darker than normal on T1-W images and brighter than normal on T2-W images. MR imaging has been used in the prenatal diagnosis of this condition in which the foetal kidneys appear enlarged and hyperintense on T2-W images. The post contrast MR images in our patient showed the striated nephrogram appearance.

\section{Hepatobiliary Manifestations/Clinical Features}

ARPKD is invariably associated with ductal plate anomalies of the biliary tree. Caroli syndrome describes dilated bile ducts along with fibrosis surrounding the portal tracts. Progressive portal fibrosis leads to portal hypertension and consequent varices and hypersplenism.(7)

Although histologic evidence of biliary involvement is universal, the clinical manifestations of hypersplenism and cholangitis are not present in all the patients and only a minority develops serious complications of portal hypertension like UGI bleed warranting portosystemic shunting or hepatic transplantation due to severe portal hypertension or recurrent cholangitis. Liver function test may remain normal or mildly elevated. Except for a single episode of hematemesis when she was 9 months old, our patient did not have any feature of hypersplenism, cholangitis and her liver function tests were normal.

\section{Radiological Features}

At US, the liver is usually normal in size with patchy or diffusely increased echogenicity. In our patient, the left and caudate lobes were increased in size with diffusely increased hepatic echotexture. Increased echogenicity of the portal tracts, representing periportal fibrosis is noted, as was noted in the case presented. The intrahepatic biliary tree may appear normal at US and CT or may be dilated. There was focal cystic dilatation of the right lobar intra-hepatic biliary radicals in Segments VI and VII in the present case. Splenomegaly is also frequently seen in patients with portal hypertension. In addition, oesophageal varices, enlarged splenic and portal veins may be demonstrated. Our patient had splenomegaly with dilated splenic vein with multiple collaterals. Doppler US interrogation of the portal and splenic 
veins may help assess the direction of flow (Hepatofugal or hepatopetal) and therefore the progression of portal hypertension.

MR cholangiography was found to be more sensitive than US in the detection of dilated bile ducts in children with ARPKD.

\section{Systemic Manifestations}

Growth impairment has been reported in children with ARPKD. It still has not been proved that growth retardation in ARPKD is indeed correlated with decreased renal function, however, like children with other forms of CKD, children with ARPKD can successfully be treated with growth hormone.(8)

The child in our case had short stature but her renal parameters were within normal limits. She was hypothyroid but except for decreased number of carpal bones for age, her skeletal radiographs did not reveal any feature of cretinism. The reason of her short stature hence was not determined in our patient.

Since the children with ARPKD often have both CKD and severe hypertension during critical early years of neurodevelopment, they may be at particular risk for worse neurocognitive outcomes. Additional studies are however needed in ARPKD patients to fully characterize their neurocognitive and behavioural functioning. Clinicians need to maintain a high index of suspicion for neurocognitive problems and provide referrals as appropriate in children with ARPKD.

In our patient, at initial presentation, she had cognitive impairment. But then, she had features of hepatic encephalopathy and on correction of this condition her mental status was clinically normal.

In addition to the hepatic, pancreatic and renal cysts, cystic lesions in the thyroid gland may be seen with ADPKD, but no documentation has been obtained regarding changes in the thyroid gland in ARPKD. Whether the ultrasonographic feature of the thyroid gland that was obtained in our patient is a co-morbidity of ARPKD-CHF, or merely a co-existing entity requires further study.

\section{Prenatal Diagnosis and Post-Natal Outcome}

Prenatally ARPKD can be first suspected based on routine obstetrics ultrasound which reveal symmetrically enlarged, echogenic kidneys with loss of corticomedullary differentiation Oligohydramnios may be present due to poor foetal urine output. Prenatal genetic testing can be performed by using haplotype analysis or PKHD1 sequencing.

\section{Post Natal Outcome}

The estimated perinatal mortality rate in ARPKD patients is $\sim 30 \%$, primarily due to respiratory compromise as a result of pulmonary hypoplasia. The enlarged kidneys may also interfere with nutrition due to gastrointestinal tract compression. Aggressive management strategies for infants with severe ARPKD have been described, including unilateral or bilateral nephrectomy to improve ventilation and nutrition.

Complications of ARPKD in the neonatal period include respiratory distress, fluid and electrolyte imbalance, oliguria, acute kidney injury and hypertension. There are no diseasespecific treatments for ARPKD, and treatment is focused on kidney disease (and its associated complications), and portal hypertension, varices, ascending cholangitis and liver failure requiring transplantation. Chronic lung disease and growth retardation are also potential complications, although these may become clinically less significant in adolescence and adulthood. Hypertension may be severe and occurs early in the course of disease and ACE inhibitors are considered the treatment of choice in the management of chronic kidney disease in children which includes monitoring and treatment of metabolic derangements (Hyperkalaemia, metabolic acidosis, anaemia, metabolic bone disease), with close monitoring and optimization of nutrition and growth. Feeding difficulties are common in paediatric patients with ARPKD, in young children due to impingement on the stomach by massively enlarged kidneys resulting in early satiety and gastroesophageal reflux, often warranting unilateral or bilateral nephrectomise. Enteral feedings via nasogastric or gastrostomy tubes are often needed to ensure delivery of sufficient calories.

Patients with ARPKD may have evidence of haematuria as with ADPKD. ARPKD patients can develop urinary tract infections which can generally be treated with standard medications. With advances in renal replacement therapy, complications of $\mathrm{CHF}$ have seen to dominate the clinical picture in adolescents and adults. Almost half of the neonatal survivors develop evidence of portal hypertension over time. Patients with CHF are at risk for life-threatening ascending cholangitis, which may occur at any time with symptoms of fever with upper quadrant pain and/or elevation of transaminases or biliary markers. Periodic endoscopy is recommended to identify and monitor the severity of oesophageal varices which may warrant oesophageal banding.

In the past, the more severe cases of portal hypertension were treated with porto-systemic shunts. However, several case studies reported the occurrence of hepatic encephalopathy in patients with shunts who subsequently progressed to end-stage renal disease.

Combined liver-kidney transplantation has also been recommended for those with CKD and significant liver involvement. ${ }^{(9)}$

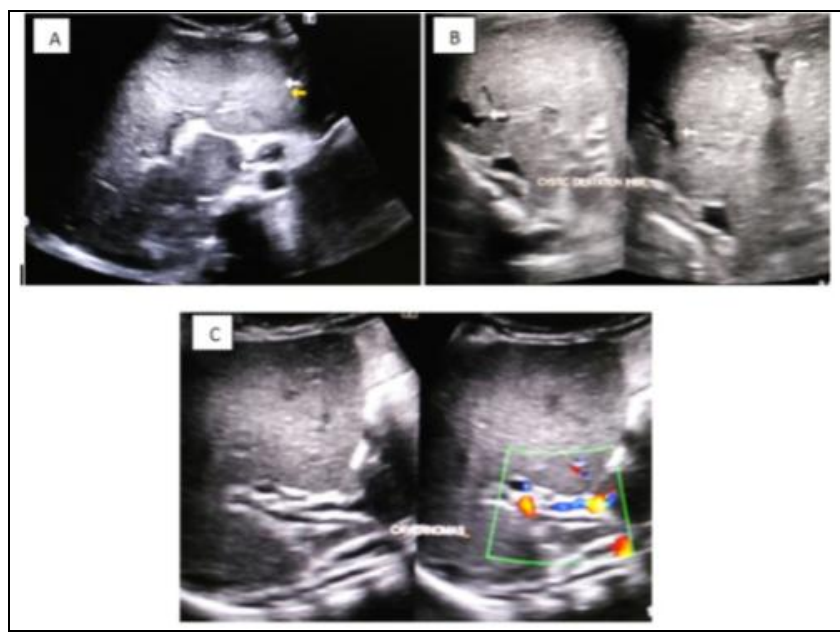

Figure 1. USG whole abdomen revealed mild hepatomegaly with enlargement of the caudate lobe $(A)$. There was cystic dilatation of the intra-hepatic biliary radicals in Segment VII of the liver (B). The portal vein showed cavernous transformation (C) 

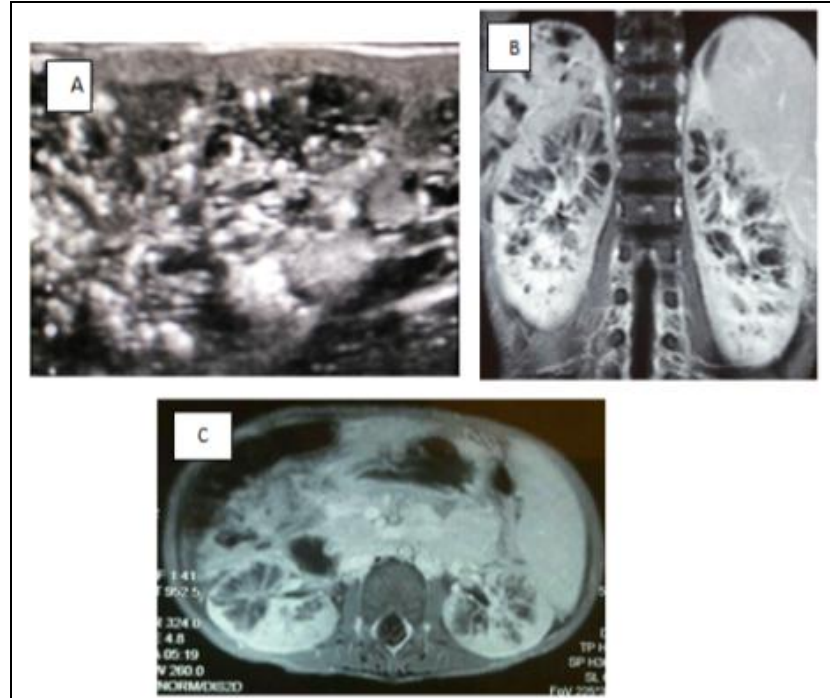

Figure 2. High resolution USG of bilateral kidneys shows increased echogenicity of the renal pyramids along with multiple hyperechoic foci casting posterior acoustic shadow. (A) MRI abdomen revealed enlarge bilateral kidneys (B) with striated nephrogram (C)

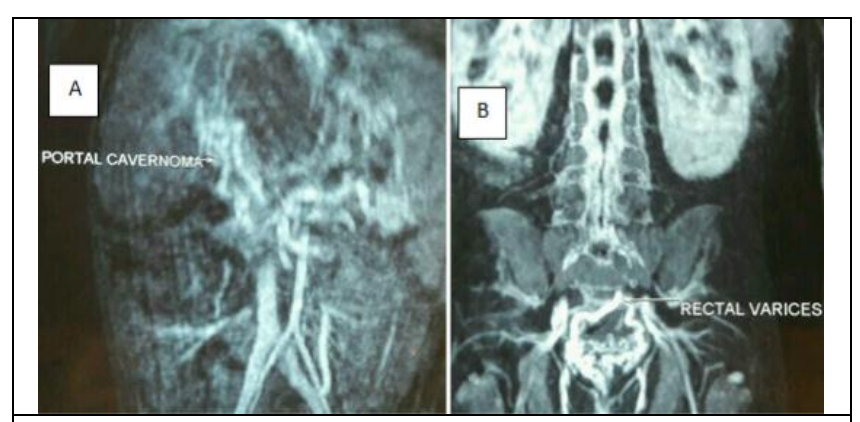

Figure 3. Portal vein showed cavernous transformation (A) along with multiple para-rectal varices (B)

\section{FINAL DIAGNOSIS}

1. ARPKD With CHF Complicated by Portal Hypertension with Cavernous Transformation of Portal Vein, Multiple Collaterals and Splenomegaly.

2. Hepatic Encephalopathy.

\section{REFERENCES}

[1] Lonergan GJ, Rice RR, Suarez ES, et al. Autosomal recessive polycystic kidney disease: radiologicpathologic correlation. Radiographics 2000;20(3):837-55.

[2] Hartung EA, Guay-Woodford LM. Autosomal recessive polycystic kidney disease: a hepatorenal fibrocystic disorder with pleiotropic effects. Pediatrics 2014;134(3):e833-e45.

[3] Patel JN, Gupta S, Fauzdar M, et al. Congenital hepatic fibrosis associated with polycystic kidney disease. J Liver 2015;4:171. doi:10.4172/2167-0889.1000171.

[4] Turkbey B, Ocak I, Daryanani K, et al. Autosomal recessive polycystic kidney disease and congenital hepatic fibrosis (ARPKD/CHF) Pediatr Radiol 2009;39(2):100-11.

[5] Wen J. Congenital hepatic fibrosis in autosomal recessive polycystic kidney disease. Clin Transl Sci 2011;4(6):460-5.

[6] Daneman A, Navarro OM, Somers GR, et al. Renal pyramids: focused sonography of normal and pathologic processes. Radiographics 2010;30(5):1287-307.

[7] Brancatelli G, Federle MP, Vilgrain $V$, et al. Fibropolycystic liver disease: CT and MR imaging findings. Radiographics 2005;25(3):659-70.

[8] Lilova M, Kaplan BS, Meyers KE. Recombinant human growth hormone therapy in autosomal recessive polycystic kidney disease. Pediatr Nephrol 2003;18(1):57-61.

[9] Dell KM. The spectrum of polycystic kidney disease in children. Adv Chronic Kidney Dis 2011;18(5):339-47. 